\title{
A Study of Bandwidth Allocation Strategies for Interactive Image Applications
}

\author{
Partho P. Mishra \\ ATET Bell Laboratories \\ Murray Bill, New Jersey
}

\author{
Satish K. Tripathi \\ Department of Computer Science \\ University of Maryland
}

\begin{abstract}
Image applications are expected to consume a significant portion of the bandwidth of future integrated service networks. In this paper, we examine the effectiveness of various bandwidth allocation mechanisms for this class of applications. First, we describe how a rate based hop by hop (HBH) congestion control scheme, proposed previously by us for controlling data traffic, may be modified to control the flow of image traffic. The performance of the modified $\mathrm{HBH}$ scheme as measured by the average image transfer times is shown to be within $15-30 \%$ of an unachievable lower bound. We also compare the effectiveness of the HBH scheme with a hierarchical round robin (HRR) scheme that reserves bandwidth before commencing each image transfer. Our results indicate that the likelihood of exceeding a desired transfer time requirement with the $\mathrm{HBH}$ scheme is lower than the blocking probability with the HRR scheme. Moreover, the average transfer times achieved with the $\mathrm{HBH}$ scheme are much lower. However, the variation of the transfer time is very small with the HRR scheme. We also show that the HBH scheme may be used to effectively trade transfer time vs image quality.
\end{abstract}

\section{INTRODUCTION}

Applications involving image transfers are expected to consume a significant portion of the bandwidth in integrated service networks. There are relatively few applications today

*This work was done when the first author was at the University of Maryland and was supported in part by TASC. 
that use networks to transfer large images primarily because the required bandwidth is either unavailable or too expensive. For example, the transfer of a 200 Mbyte cat scan image over a $1.5 \mathrm{Mb} / \mathrm{s}$ leased line would take more than 25 minutes - an unacceptably large latency. Similarly, it is currently cheaper to transfer the high quality photographic images used in magazine publishing using courier services. However, the advent of high speed integrated service networks and greater accessibility to network resources promises to drastically alter this situation. There are an increasing number of applications, e.g. IMM [Dan], that provide various kinds of image access services and this trend is likely to accelerate.

The service quality requirements of image applications are in some ways similar to that of data traffic. For example, the major metric of performance is the transfer time: the time taken to transfer individual images or sets of images. This value is determined by the average throughput seen by a connection. Hence, as with bulk data transfer traffic, it is desirable to provide as much throughput as possible to individual connections. However, unlike data traffic the redundancy in information in each image implies that some amount of loss may be tolerable. Additionally, under circumstances where the bandwidth available is limited it is possible to transmit the information at a coarser level of granularity to trade image quality for transfer time latency.

There are two choices available for allocating bandwidth to image applications transported over a packet switched wide area network. One possibility is to allocate a fixed bandwidth to a connection, based on a quality of service contract between the application and the network. The other possibility is to adapt the bandwidth used by an application depending on available network capacity, as with data traffic applications [Jac88;RJ90]. However, the adaptive rate control mechanisms designed for data traffic applications need to be modified to reflect the differences in the service quality requirements of the two classes of traffic.

In this paper, we examine the effectiveness of various bandwidth allocation schemes for image traffic. First, we discuss how a rate based hop by hop (HBH) congestion control scheme, previously proposed by us to control data traffic [MK92;MKT93], may be modified to control the flow of image traffic. We compare the performance of the modified HBH scheme as measured by the average transfer times with an unachievable lower bound on the transfer time and show that the average transfer times achieved with the $\mathrm{HBH}$ mechanism are within $15-30 \%$ of the lower bound. Second, we show that allocating bandwidth to aggregates of connections results in superior performance specially in counteracting the degradation in the performance of adaptive control schemes as the transfer times become smaller vis-a-vis the end to end delay in the network. Third, we compare the effectiveness of adaptive and fixed bandwidth allocation using the HBH and Hierarchical Round Robin (HRR) scheduling schemes [KKK90] as examples. Our results indicate that the likelihood of exceeding a desired transfer time requirement with the HBH scheme is lower than the 
blocking probability with the HRR scheme. Moreover, the average transfer times are much lower when the HBH scheme is used. However, the variance of the transfer time is very low with the HRR scheme.

The rest of this paper is organized as follows. In section 2 we describe digital image representation and coding techniques as well as the traffic characteristics and the service qulaity requirements of typical applications. In Section 3 we describe the different congestion control mechanisms that we evaluate. In Section 4 we describe the experimental methodology we use to evaluate the performance of the control schemes and present the results. Section 5 concludes the paper.

\section{APPLICATION CHARACTERISTICS}

The digital representation of an image is a two dimensional array of pixels. The number of bits required to represent every pixel depends on the degree of resolution and whether or not color is used. The simplest way to represent an image is by explicitly storing the bits corresponding to every pixel in the image. However, this requires a huge number of bits to represent an image - for example a single $25000 \times 25000$ pixel image with 8 bits per pixel would require 5 Gigabits. The number of bits required to represent an image may be reduced by using compression techniques. Compression techniques achieve reduction in two ways. The first is by the use of a more efficient encoding scheme in which the representation for a symbol (a pixel value) is chosen based on how frequently it occurs in the image. Examples of these techniques include Huffman coding and arithmetic coding [NP85]. The second is by discarding information which is less relevant to image quality by quantization. The achievable compression and the loss in information varies depending on coding algorithm and the contents of an image. Existing compression algorithms can result in a reduction in the number of bits required to encode an image by a factor 10:1 to 50:1, without a significant reduction in the image quality. Due to the potentially large size of images, many applications also operate on smaller portions of an image, which we will refer to as tiles. In this paper we will assume that a tile is $256 \times 256$ pixels.

Most image transfer applications send or receive information in either a continuous or interactive mode. In a continuous mode transfer, a set of images is transferred from an image repository to a group of recipients. An example of such an application is the transmission of satellite weather images to meteorological bureaus. In an interactive mode transfer, image transfers proceed in cycles with a user requesting a few images or parts of an image, viewing/analyzing the images retrieved and then requesting more images. An example of such an application is image browsing where a user browses through an image database with images being transmitted on a demand basis. The main difference between the two categories of applications is that for continuous mode transfers, a much larger amount of information is transferred between idle periods and the duration of these idle 
periods is also much longer.

Applications belonging to the first category typically require a fixed level of quality and transmit large amounts of information. Hence, the traffic characteristics and service quality requirements of these applications is very similar to that of bulk data transfers. Thus the insights derived from the study of congestion control mechanisms for data traffic apply to this class of applications as well. However, for the second category of applications the quality may be changed dynamically depending on the service quality requirements of the user and the network load and the transfer times are relatively small. Hence, in this paper we have chosen to focus on the problem of how control mechanisms should be designed to handle this class of interactive application. We model the traffic characteristics of this class of applications using a client server communication model. We assume that clients issue image transfer request messages from time to time. In response to these messages, a server commences an image transfer. Thus a client is either idle, corresponding to the time during which the user analyzes the image, or waiting for an image transfer to finish. The start of the idle period starts from the instant at which the image transfer completes. This model is based on empirical studies of various interactive image applications such as image browsing [Bra].

The service quality requirements of image applications is dependent both on the nature of the applications as well as the specific operations being performed. As we have already indicated there are two principal metrics of service quality: transfer time and image quality. Desirable transfer times should be less than 2000-3000 ms for the image fetches. The desired image quality typically varies depending on the user's preferences.

\section{CONTROL MECHANISMS}

For the class of image applications modeled, an image server needs to transfer a certain number of tiles in response to client requests. These tiles could correspond to a part of an image, an entire image or a set of images. The number of bytes required to represent the image depends on the desired quality $q$ and the information content of the image $I$ : we denote this as $f(q, I)^{1}$. Let the minimum and maximum desired image quality be $Q_{\min }$ and $Q_{\max }$, respectively, and let the maximum desired transfer time be $T$. How should the server decide at what rate to send packets to the client? There are two possibilities. The first possibility is to decide a fixed sending rate based on bandwidth availability and the transfer parameters: $T, Q_{\max }, Q_{\min }$ and $f(q, I)$. In this case the quality stays constant for the duration of the transfer. Alternatively, the server can send packets at a variable rate with sending rate being updated periodically based on network conditions. The sending rate adaptation may be achieved by either increasing the transfer time or by reducing the image quality. In the second case, the quality may (potentially) be altered every time the

\footnotetext{
${ }^{1}$ Since there may be more than one way to adjust image quality, $q$ is a composite measure.
} 
sending rate is recomputed. For both the fixed and adaptive rate techniques it is possible to tradeoff the image quality with the time taken to complete the transfer.

\subsection{Rate Reservation}

The fixed rate approach to provide service quality guarantees is based on either explicit or implicit bandwidth reservation. With explicit reservation, a scheduling mechanism is used at each switch to ensure that individual connections get a certain guaranteed level of bandwidth [Gol91; KKK90; Zha91]. In the case of implicit reservation, admission control mechanisms are used to ensure that the arrival rate of packets for all connections does not exceed the available bandwidth [Bou92]. However, the scheduling mechanism used is simple, typically something like FCFS. Since the use of explicit reservation isolates connections from each other, it can provide better service quality guarantees. Hence, for the experiments described in this paper we choose a explicit reservation policy, using the HRR scheduling mechanism to allocate bandwidth at switches [KKK90].

The HRR scheduling discipline guarantees a minimum service rate to a connection independent of the traffic generated by other connections. In conjunction with an admission control mechanism, this discipline allows clients to get performance guarantees in terms of throughput, delay, delay jitter and loss rate. In this scheme, transmission on the output link of a switch occurs in rounds of a fixed time duration. The parameters used to define the rate for a connection are the quantum which defines the number of packets that can be sent per round and the interval which defines the number of rounds to skip when the rate of the connection is less than one packet per round. The reader is referred to [KKK90] for a more complete description of the mechanism and possible implementation strategies.

For the image applications that we model, the explicit reservation approach involves choosing a value of the desired bandwidth and reserving it at each switch on the route every time an image transfer takes place. The reserved bandwidth is freed as soon as the image transfer is complete. We assume that a client sends in an image transfer request over an unguaranteed connection. When the server receives such a request from a client, it sends out a bandwidth reservation request packet specifying a set of acceptable rates $R$. We assume that these rates are calculated as:

$$
R=\frac{f(q, I)}{T}, \forall q \in\left(Q_{\min }, Q_{\max }\right)
$$

If any of the intermediate switches cannot provide even the minimum requested rate, the server waits for a fixed interval of time, $t_{w}$, and then generates a new request. The new rates requested are computed using $T-t_{w}$, rather than $T$, in Equation 1. A later request may succeed because of extra bandwidth becoming available in the network due to the 
termination of other image transfers. The server keeps sending requests until it succeeds or the transfer time requirements are exceeded. In the latter case the image transfer blocks, as a result of which the client is assumed to abort the request and send a new request after the usual idle period.

\subsection{Rate adaptation}

The adaptive rate based approach attempts to optimize the transfer time by adjusting the sending rate based on the network load. The control mechanisms used for adapting the flow of image traffic may be derived from those used for adapting the flow rate of data traffic with suitable modifications. The most commonly used congestion control mechanisms for adjusting the flow of data traffic are the JK and the DECbit schemes [Jac88; RJ90]. Our previous work [MK92; KKM93] suggests that the HBH control mechanism performs much better than either of these schemes in high bandwidth delay product networks. Hence, we choose the $\mathrm{HBH}$ control mechanism as a representative scheme to test the performance of adaptive control mechanisms for adjusting the sending rate. However, we modify the scheme in the following manner to allow the sender to reduce image quality to obtain lower transfer times.

The HBH control mechanism computes the sending rate for a connection at each host/switch on the path of the connection. The sending rates of connections are dynamically adjusted at a switch, using feedback information provided by the neighbouring switches. The desired service rate is computed using a control equation: the goal being to optimize the transfer time for individual connections by trying to get as much throughput as possible while avoiding excessive packet losses. The control equation attempts to keep the queue occupancy for each connection, at the downstream, at a target level. We refer to this value as the setpoint. We refer the reader to [MK92; MKT93] for greater details about the control mechanism.

We modify the HBH mechanism to control the flow of image traffic in the following manner. The sender computes the estimated completion time of an image transfer every time it recomputes the sending rate. If we denote the estimate of the bandwidth at the host (sender) at time $t$ as $R(t)$ and the number of bytes remaining to be transferred as $f^{\prime}(q, I, t)$, the estimated completion time $(F)$ at a particular quality level $q$, may be computed as

$$
F=t+\frac{f^{\prime}(q, I, t)}{R(t)}
$$

Based on the above computation, the application picks the highest quality level which will enable the transfer to complete within the desired time. We assume that the changes in the quality level are done on a per-tile basis. 
We also explore a version of the HBH scheme where the sending rate of an aggregate group of connections, rather than only a single connection, is adjusted. In this scheme it is assumed that all packets belonging to the same aggregated group are queued together at network switches and hosts and sent out in a First-In-First-Out manner. A rate control mechanism is enforced in the network layer protocol at the host and switches as described in [MK92; MKT93]. This control mechanism forces packets to be sent out from the common queue (at the host/switch) to the downstream switch at a particular rate. This rate is dynamically updated based on periodic feedback messages from the neighbouring switches. The bandwidth available to an individual connection depends on its share of the total output rate for the group to which it belongs. The transport layer protocol (for an individual connection) maintains an estimate of this bandwidth and picks the quality level using Equation 2.

In the experiments described in this paper, we assume completely reliable information transfer, although in practice it may be possible to tolerate some packet losses. To provide reliable transmission, we use a transport protocol which uses block based acknowledgements and selective retransmissions. A block consists of a fixed number of packets. We assume that a fixed number of blocks can be in transmission simultaneously. This is analogous to the usual concept of a window. Each packet is identified by a block number and an offset number defining its position within the block. The sender transmits all the packets within a block marking the last packet so as to force the receiver to send an acknowledgement. A timer is set when the last packet in a block is sent. A receiver maintains a $B$ bit-vector, where $B$ is the block size. When a packet is received the corresponding bit is set in the bit-vector. The acknowledgment packet sent by a receiver contains the number of the block being acknowledged and the bit vector for that block. By examining the acknowledgments from each receiver, the sender identifies lost packets and schedules them for retransmission. If no packets are lost, the sender advances the block window. If the timer goes off before an acknowledgment is received then all the packets in the block are retransmitted.

\section{EXPERIMENTAL SETUP AND RESULTS}

In this section, we evaluate the performance of the HBH and HRR schemes for image transport. We first study the performance of the $\mathrm{HBH}$ mechanism when used to control the sending rate of individual connections, assuming that quality changes are not allowed once a transfer commences. We next study the performance of the $\mathrm{HBH}$ mechanism when it used to control the sending rate of aggregates of connections. We then compare the performance of the HBH and HRR mechanisms. Finally we evaluate how well the HBH mechanism can be used to allow a transfer time vs image quality tradeoff.

We use discrete event simulations to evaluate the effectiveness of the control mech- 
anisms. The traffic generated by a connection is created using the analytical models described in Section 2. We assume that the idle periods for an application are distributed exponentially with a mean of 30 seconds. We also assume that each of the applications switches on $n$ secs from the start of the simulation, where $n$ is an exponentially distributed random variable with a mean of 15 seconds. These values were chosed based on empirical measurements of image applications [Bra]. We use a very large number of connections to capture the effect of the aggregate traffic arrival process at a switch. We remark that it is not possible to achieve this type of aggregation by using an analytical model for the aggregate arrival process (created by a superposition of the arrival process for individual connections) because the traffic arrival process for a connection is partially dependent on the congestion control mechanism that is used. The experimental topology used is shown in Figure 1. This topology is chosen to capture a typical fan-in topology where a powerful image server handles multiple clients. The speed of the ingress links from each of the hosts to the switches $(200 \mathrm{Mb} / \mathrm{s})$ is deliberately chosed to be faster than the speed of the backbone link ( $50 \mathrm{Mb} / \mathrm{s})$ between Switches 1 and 2 so that the backbone link is the bottleneck.

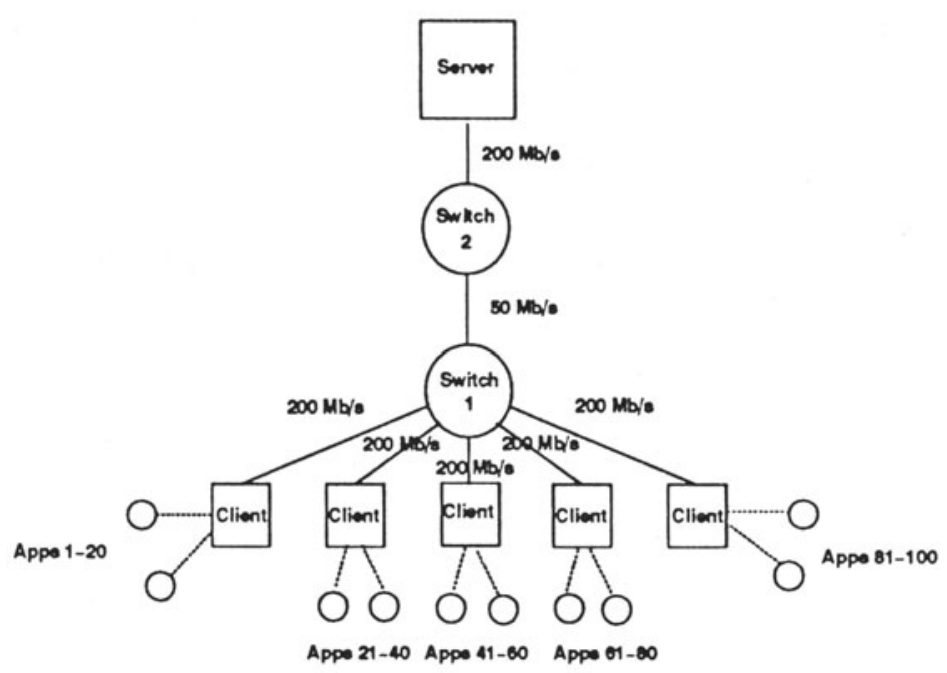

Figure 1: Simulation configuration

We used fixed size packets of 500 bytes. The duration of each simulation run is 2000 
Table 1: Comparison of HBH and OPT

\begin{tabular}{|c|c|c|c|c|c|}
\hline & Buffers & Number of conns & Transfer time & Utilization & Loss rate \\
\hline HBH & 400 & 100 & $1406 \mathrm{~ms}$ & .850 & $1.53 \%$ \\
\hline HBH & 1000 & 100 & $1237 \mathrm{~ms}$ & .864 & $0.35 \%$ \\
\hline OPT & $\infty$ & 100 & $1124 \mathrm{~ms}$ & .871 & - \\
\hline \hline HBH & 400 & 80 & $774 \mathrm{~ms}$ & .714 & $0.40 \%$ \\
\hline HBH & 1000 & 80 & $749 \mathrm{~ms}$ & .717 & $0.00 \%$ \\
\hline OPT & $\infty$ & 80 & $583 \mathrm{~ms}$ & .719 & - \\
\hline
\end{tabular}

seconds ${ }^{2}$ so that the confidence intervals for the mean measures are less than $10 \%$ of the sample mean.

The main goal of a rate adaptation mechanism, such as $\mathrm{HBH}$, is to trade off the losses seen by a connection against the achievable throughput. A very conservative policy results in reduced utilization of the bottleneck bandwidth which leads to an increase in the overall transfer time. On the other hand, a very aggressive policy leads to excessive packet losses necessitating retransmissions with a concomitant increase in the overall transfer time. Hence the most useful metric that may be used to measure the performance of such control mechanisms is the transfer time ${ }^{3}$. This measure subsumes both transient and steady state performance. As we have pointed out, this is also one of the primary service quality metrics for image applications. To measure how well a rate adaptation mechanism performs, we compute a lower bound on the transfer time. This (unrealizable) lower bound corresponds to a situation where there is an infinite amount of buffering available at each node and each connection injects data into the network at the speed of the access link connecting the host to the first switch on the path of the connection. For simplicity, we will refer to this as the optimal scheme.

In the first set of experiments we compare the performance of $\mathrm{HBH}$ control with the optimal scheme. The topology used for the experiments is shown Figure 1 with either 400 or 1000 buffers at the switches. There are either 80 or 100 active connections and the traffic arrival process for each connection is generated as described previously. The time instant at which a new request is generated depends on when the previous transfer was completed. As a result, the average utilization depends both on the control scheme as well as the number of active connections. For the optimal scheme, the average utilization is about $72 \%$ and $87 \%$ with 80 and 100 connections respectively.

Table 1 shows the average transfer times, loss rates and the average utilization for this

\footnotetext{
${ }^{2}$ corresponding to about $2,500,000$ packets going through the bottleneck link

${ }^{3}$ We will occasionally describe this as the actual transfer time to distinguish from the target transfer time
} 
Table 2: Impact of aggregation

\begin{tabular}{|c|c|c|c|c|}
\hline & Buffers & Number of conns & Transfer time & Loss rate \\
\hline HBH & 400 & 100 & $1406 \mathrm{~ms}$ & $1.53 \%$ \\
\hline HBH & 1000 & 100 & $1237 \mathrm{~ms}$ & $0.35 \%$ \\
\hline Aggr & 400 & 100 & $1146 \mathrm{~ms}$ & $0.12 \%$ \\
\hline \hline HBH & 400 & 80 & $774 \mathrm{~ms}$ & $0.40 \%$ \\
\hline HBH & 1000 & 80 & $749 \mathrm{~ms}$ & $0.001 \%$ \\
\hline Aggr & 400 & 80 & $712 \mathrm{~ms}$ & $0.14 \%$ \\
\hline
\end{tabular}

set of experiments. The results indicate that the use of the $\mathrm{HBH}$ control mechanism results in transfer times that are about $10-30 \%$ greater than the optimal. The greater transfer times may be explained as follows. In the $\mathrm{HBH}$ control mechanism each connection linearly increases its sending rate when it starts a new transfer. For the application we model, the duration of a single transfer is relatively short lived. As a result the average throughput seen by a connection, when only a few connections are active, is not as high as it could be with a more aggressive policy.

Figures 2 and 3 which show the throughput for a single image transfer for a particular connection, controlled using either $\mathrm{HBH}$ or the optimal scheme, illustrates this point. Figure 3 shows the cumulative distribution of the average throughput. Note that the maximum throughput for a $\mathrm{HBH}$ controlled connection never exceeds $31 \mathrm{Mb} / \mathrm{s}$ while with the optimal policy a throughput of up to $50 \mathrm{Mb} / \mathrm{s}$ could be obtained ${ }^{4}$.

The results also indicate (as might be expected) that a greater number of buffers leads to fewer losses and retransmissions. With the HBH scheme, it is possible to adjust the loss-throughput tradeoff seen by a connection by adjusting the parameters of the control mechanism. In our experiments we use an error control mechanism to guarantee totally reliable transmission. However, since totally reliable transmission may be avoided for certain types of image applications, it is possible to further reduce the average transfer time when using the $\mathrm{HBH}$ scheme using a less conservative error control policy.

The previous experiment indicated that sub-optimal performance is due to the relatively small transfer times. One way to reduce the average transfer time is to control aggregates of connections rather than individual connections. The idea here is that controlling aggregates of connections increase the effective lifetime of an aggregated connection, thereby reducing the impact of the linear probe-up phase on the average transfer time. Table 2 compares the effect of aggregated control with per connection control. For

\footnotetext{
${ }^{4}$ Even for optimal control a throughput of $50 \mathrm{Mb} / \mathrm{s}$ is never obtained because the transfer time used for computing the average throughput includes the time taken to send the request from the client to server.
} 


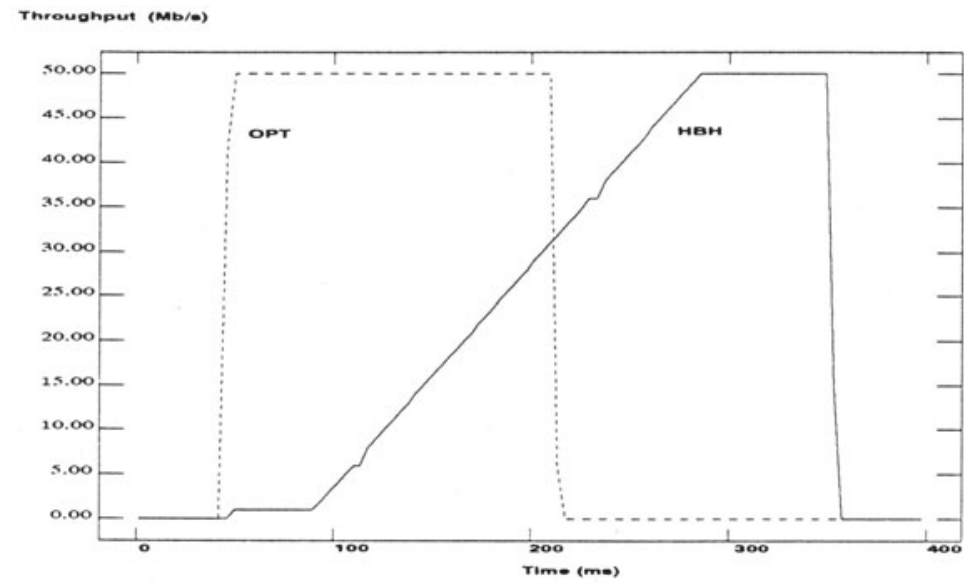

Figure 2: Instantaneous throughput for a single image transfer with $\mathrm{HBH}$ and OPT

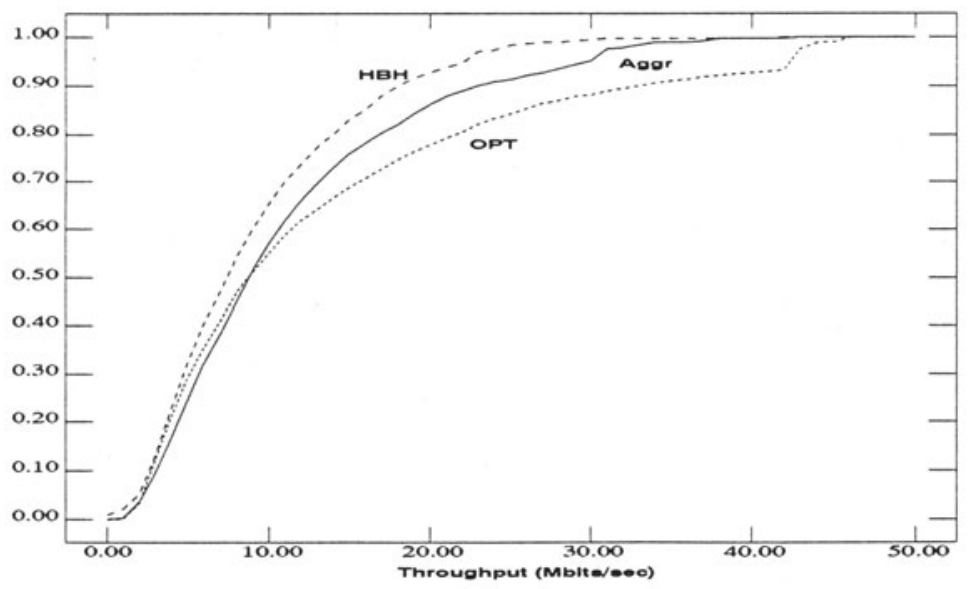

Figure 3: Cumulative distribution of throughput with HBH, Aggr and OPT 
Table 3: Comparison of HBH and HRR, QBP: Quasi blocking probability percentage, BP: Blocking probability percentage.

\begin{tabular}{|l|c|c|c|c|c|}
\hline & Conns & Transfer time & Utilization & QBP & BP \\
\hline HBH(2s) & 100 & $1268 \mathrm{~ms}$ & .839 & 14.37 & - \\
\hline HBH(3s) & 100 & $1268 \mathrm{~ms}$ & .839 & 4.71 & - \\
\hline HRR(2s) & 100 & $2062 \mathrm{~ms}$ & .676 & - & 14.68 \\
\hline HRR(3s) & 100 & $3059 \mathrm{~ms}$ & .706 & - & 9.55 \\
\hline \hline HBH(2s) & 80 & $774 \mathrm{~ms}$ & .714 & 2.04 & - \\
\hline HBH(3s) & 80 & $774 \mathrm{~ms}$ & .714 & 0.11 & - \\
\hline HRR(2s) & 80 & $2062 \mathrm{~ms}$ & .610 & - & 7.30 \\
\hline HRR(3s) & 80 & $3059 \mathrm{~ms}$ & .616 & - & 3.54 \\
\hline
\end{tabular}

the same amount of buffering, aggregated control clearly results in much better performance than individual control.

\subsection{Comparison with rate reservation}

The main performance metric for reservation based approaches is the blocking probability which is the likelihood that a user requested transfer does not complete. For the modeled application, this value is dependent on the target transfer time and image quality requested for a particular transfer. For an adaptive bandwidth allocation scheme the main performance metric is the average value of the (actual) transfer time. To be able to compare the two types of schemes, we define a quasi blocking probability for an adaptive scheme as the likelihood that the actual transfer time will exceed the target transfer time.

Table 3 compares the blocking and quasi blocking probabilities for HRR and HBH for a target transfer time value of either 2 or 3 seconds and for a fixed level of quality. There are 400 buffers at the switch and either 80 or 100 connections. We also show the average utilization of the bottleneck link and the average transfer time. Figure 4 shows the cumulative distribution of the transfer time. The results show that the average transfer times with $\mathrm{HBH}$ are much lower ${ }^{5}$ while the variation in the transfer time is much smaller with HRR. As the target transfer time is increased for the HBH scheme, the value of QBP decreases because the number of transfers whose duration exceeds the target transfer time value decreases. For example, Figure 4 shows that 2.04 /only $0.11 \%$ take more than 3 seconds. More interestingly, the results show that the quasi blocking probability with HBH is lower than the blocking probability with HRR. This may be explained by the

\footnotetext{
${ }^{5}$ In general the ratio of the average time with HRR to that with HBH will tend towards $\mathrm{n}: 1$ as the load tends to zero.
} 
following three factors. First, with HRR, connections are assigned rates in multiples of a set of basic rates. As a result, there may be an effect similar to fragmentation in memory allocation, with image transfers being blocked even though the utilization is less than $100 \%$. This is why the average utilization with HRR is less than with $\mathrm{HBH}^{6}$.

The second factor that contributes to a higher blocking probability for HRR is that the bandwidth at a switch can stay reserved but unutilized for up to 2 end to end round trip times due to the bandwidth reservation and release operations. Finally, with HBH control each connection finishes an image transfer faster, on the average, than with HRR. Consequently, when many connections commence image transfer over a relatively small time interval, the number of contending connections is lower with $\mathrm{HBH}$ control.

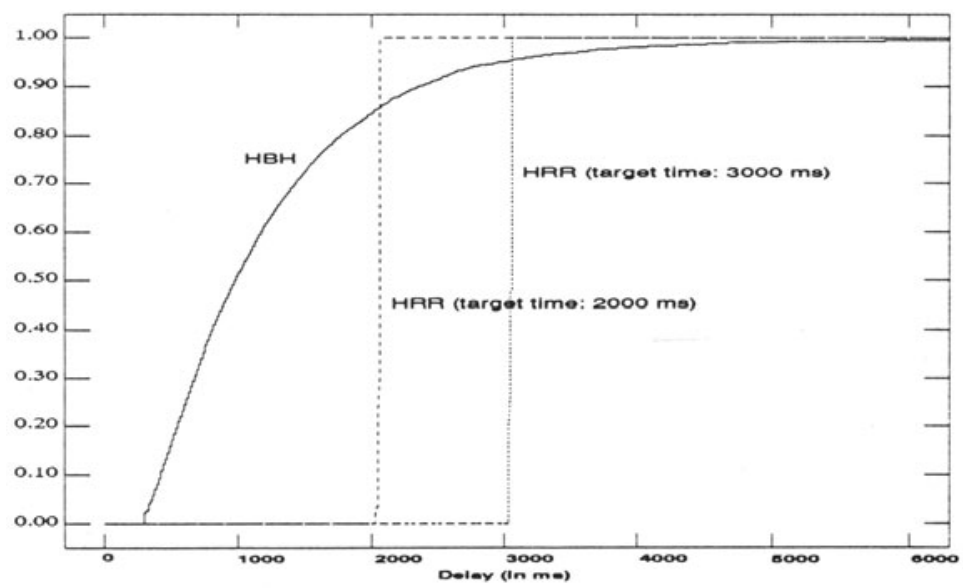

Figure 4: Comparison of $\mathrm{HBH}$ and HRR in terms of cumulative distribution of transfer times

\subsection{Handling quality changes}

For situations where an application needs the ability to trade image quality for lower transfer times, it is possible to use the $\mathrm{HBH}$ scheme with the sending rate being adjusted according to Equation 2. To investigate the effectiveness of this scheme, we look at the average quality, average transfer time and the quasi blocking probability for different values of the target transfer times at various loads. We assume that a set of 4 prerecorded streams corresponding to four levels of quality $(0,1,2$ and 3 , with 0 representing

\footnotetext{
${ }^{6}$ Another reason for the lower utilization is that the average transfer time with HRR is greater and as a result the average arrival rate is slightly lower.
} 
best quality) is available. We assume that the average compression ratio at each of these levels is $1: 1,1: 4,1: 10$ and 1:50 respectively.

The results for this set of experiments is summarized in Table 4. Figure 5 shows the cumulative distribution of the transfer time for 100 connections for a target transfer time of 1,2 and 3 seconds with 400 buffers. The quality value is computed as the mean of the quality of each individual tile that is transferred. The closer this value is to 0 , the better the quality. These results show that the HBH scheme provides the ability to smoothly trade between the image quality and the transfer time. However, there is a non-negligible probability of exceeding the target transfer time which increases with the load. The main reason for this violation is due to the retransmission of lost packets, usually resulting from a timeout. Since the maximum timeout interval used in our experiments is 250 milliseconds, most of the transfers that exceed the target transfer time, exceed it by less than 250 milliseconds. This can be observed in Figure 5 .

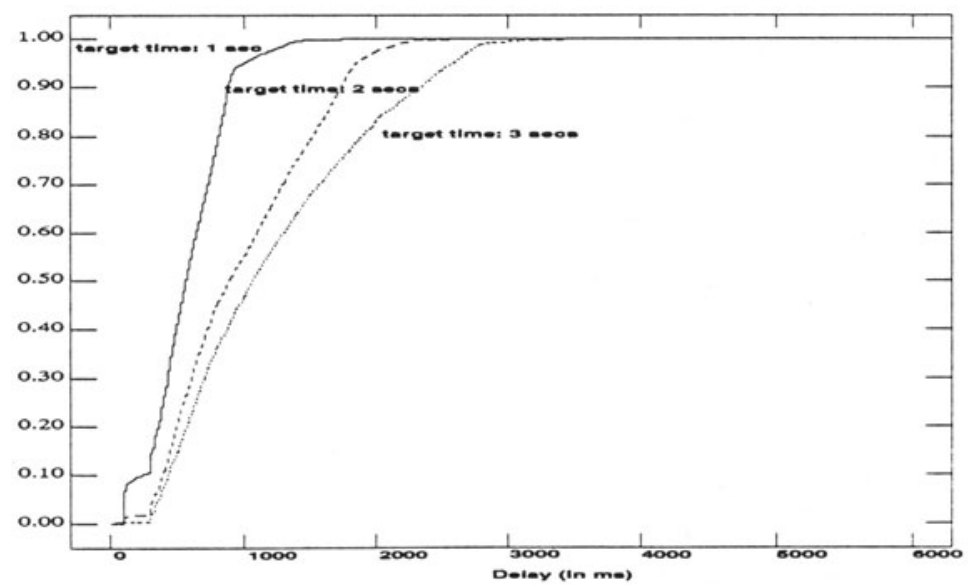

Figure 5: Cumulative distribution of the transfer time with HBH with variable quality

\section{SUMMARY}

In this paper, we have examined the effectiveness of various bandwidth allocation schemes for interactive image traffic applications. We have described how a hop by hop congestion control mechanism, proposed to control the flow of bursty data traffic, may be modified to control the flow of image traffic. The modified scheme allows a user to smoothly tradeoff the image quality against the transfer time. We have shown that for a fixed level of 
Table 4: Effect of allowing quality changes

\begin{tabular}{|c|c|c|c|c|}
\hline Conns & \multicolumn{3}{|c|}{ Transfer time Quality } & QBP \\
\hline & Target & Actual & & \\
\hline 120 & $1000 \mathrm{~ms}$ & $556 \mathrm{~ms}$ & 0.71 & 5.14 \\
\hline 120 & $2000 \mathrm{~ms}$ & $1275 \mathrm{~ms}$ & 0.19 & 5.00 \\
\hline 120 & $3000 \mathrm{~ms}$ & $1761 \mathrm{~ms}$ & 0.09 & 1.03 \\
\hline 100 & $1000 \mathrm{~ms}$ & $576 \mathrm{~ms}$ & 0.45 & 4.94 \\
\hline 100 & $2000 \mathrm{~ms}$ & $989 \mathrm{~ms}$ & 0.12 & 2.74 \\
\hline 100 & $3000 \mathrm{~ms}$ & $1228 \mathrm{~ms}$ & 0.05 & 0.49 \\
\hline 80 & $1000 \mathrm{~ms}$ & $526 \mathrm{~ms}$ & 0.33 & 2.19 \\
\hline 80 & $2000 \mathrm{~ms}$ & $745 \mathrm{~ms}$ & 0.08 & 1.19 \\
\hline 80 & $3000 \mathrm{~ms}$ & $826 \mathrm{~ms}$ & 0.003 & 0.05 \\
\hline
\end{tabular}

QBP: Quasi blocking probability percentage.

image quality, the proposed scheme results in average transfer times which are close to optimal. We have observed that performance degrades as the image transfer times become smaller vis-a-vis the end to end delays. This may be countered by controlling aggregates of connections rather than individual connections; we have shown that this results in a significant improvement in performance. We have also studied the feasibility of using a rate reservation based approach for image transport. In our experiments, the blocking probability for a given target transfer time with rate reservation scheme is higher than the equivalent probability for the $\mathrm{HBH}$ scheme, even at high loads. These results suggest that adaptive rate schemes are more suitable for interactive image traffic applications.

In this paper, we have assumed that the quality is a linear function of the available bandwidth. However, this relationship is general non-linear. Moreover, the benefit derived by a user depends on the perceptual quality of the images transferred. As a result the choice of the control mechanism is likely to have a direct effect on the user perceived quality. For example, damping the changes in the quality so that there is gradual change in quality over contiguous tiles may result in better user perceived performance. We expect to examine this interaction in greater detail in future work.

The results of this paper demonstrate that aggregation is an effective way to control short bursts of traffic. In the simulations presented in this paper, we have assumed that aggregates always share the same end to end paths. It is important to study how aggregated control can be achieved in practice under conditions where connections are being set up and torn down rapidly and where it may not always be possible to have a large number of connections sharing the same end to end path. We intend to look at this issue in the future. 


\section{REFERENCES}

[Bou92] J. Le Boudec. The Asynchronous Transfer Mode: a tutorial. Computer Networks and ISDN Systems, 24:279-309, December 1992.

[Bra] R. Braudes. Personal communication.

[Dan] W. Dang. Imm version 2.7. Announced on rem-conf mailing list 12-2-93. Available for anonymous $\mathrm{ftp}$ from $\mathrm{ftp}$.hawaii.edu in $/ \mathrm{ftp} /$ paccom/imm-2.7.

[Gol91] S.J. Golestani. A framing strategy for congestion management. IEEE Journal on Selected Areas in Communications,9(7):1064-1077, September 1991.

[Jac88] V. Jacobson. Congestion avoidance and control. In proceedings of ACM SIGCOMM, Stanford, California, August 1988.

[KKK90] C.R. Kalmanek, H. Kanakia, and S. Keshav. Rate controlled servers for very high-speed networks. In Proceedings of the Conference on Global Communications (IEEE GLOBECOM), San Diego, California, December 1990.

[KKM93] H. Kanakia, S. Keshav, and P.P. Mishra. A comparison of congestion control schemes. In Proceedings of the Fourth Annual Workshop on Very High Speed Networks, Baltimore, Maryland, March 1993.

[MK92] P.P. Mishra and H. Kanakia. A hop by hop rate based congestion control scheme. In Proceedings of ACM SIGCOMM, Baltimore, Maryland, August 1992.

[MKT93] P.P. Mishra, H. Kanakia, and S.K. Tripathi. Design and analysis of a hop by hop congestion control mechanism. Submitted to IEEE/ACM Transactions on Networking, 1993.

[NP85] A. Netravali and B. Prasada, editors. Visual communications Systems. IEEE Press, 1985.

[RJ90] K.K. Ramakrishnan and R. Jain. A binary feedback scheme for congestion avoidance in computer networks. ACM Transactions on Computer Systems, 8(2):158-181, May 1990.

[Zha91] L. Zhang. Virtual clock: A new traffic control algorithm for packet switching networks. ACM Transactions on Computer Systems, 9(2):101-125, May 1991. 